\title{
Spatial disorientation in right-hemisphere infarction: a study of the speed of recovery
}

\author{
JD MEERWALDT \\ From the Department of Neurology, University Hospital Dijkzigt, Rotterdam, The Netherlands
}

SUMMARY Sixteen patients with an infarct in the posterior region of the right hemisphere were tested at fixed intervals after a stroke ( 2 weeks, 6 weeks, 3 months, 6 months, 1 year) with the rod orientation test and the line orientation test. All patients initially showed spatial disorientation on the rod orientation test, while only three had a defective performance on the line orientation test. The recovery on the rod orientation test was parallel with the neurological improvement. Recovery mainly took place in the first six months after the stroke. Most patients then performed at a normal level. A relation between the size of the lesion (assessed from CT scans) and the speed of recovery was found.

Noticing that recovery from spatial disorientation did occur in the majority of patients with an infarction in the posterior region of the right hemisphere one year after a stroke, ${ }^{1}$ I tried to determine the time course of this recovery by examining a group of patients at fixed intervals. In addition, I studied the relationship between the size of the lesion and the speed of recovery. Finally, it seemed worthwhile to compare the serial results of the rod orientation test with those of the line orientation test developed by Benton et al. ${ }^{2}$

\section{Methods}

\section{Subjects}

Sixteen patients with damage of the posterior region of the right hemisphere were examined. They all had a cerebral infarct without a midline shift as assessed by CT scanning. Eight patients had a pre- and post-rolandic extension of the lesion, and eight had an exclusively post-rolandic lesion (table 1). Each group consisted of five males and three females, the ages ranging from 42 to 70 years, mean 58.2 years. The neurological signs of the patients were recorded (table 2). All patients were right-handed. The control group consisted of 40 patients ( 26 males, 14 females) visiting the outpatient department without history or signs of brain disease but suffering from disc lesions, lumbago, or mononeuropathy. The ages ranged from 18 to 72 years (mean $47 \cdot 5$ years).

Address for reprint requests: JD Meerwaldt, Department of Neurology, University Hospital Dijkzigt, Dr. Molewaterplein 40, 3015 GD Rotterdam, The Netherlands.

Received 4 June 1982 and in revised form 29 November 1982. Accepted 9 December 1982

\section{Testing procedure}

The methods used were the rod orientation test as previously employed by de Renzi et $a l^{3}$ and the line orientation test. ${ }^{2}$ All patients were tested at fixed intervals after the stroke, namely 2 weeks, 6 weeks, 3 months, 6 months and 1 year. Both the visual and the tactile part of the rod orientation test were carried out by the brain-damaged patients with the hand on the side of the cerebral lesion. In the visual part of the test the patient was not allowed to touch the rods of the model and he was requested to put those of the other pair (copy) in the same position. In the tactile part of the test the patient was blindfolded and had to estimate the spatial position of the rods of the model by palpation; otherwise the instructions were identical. No time limit was set. The patient was permitted to go from the model to the copy and back again as often as he wished. The order of presentation of the two parts of the test alternated from patient to patient. In both the visual and the tactile part of the test, the patients were first given five trials with the model placed on the side of the lesion and then five trials with the model placed on the other side, each time with the rods in a different position. After each trial the differences between the copy and the model were measured in the horizontal and the vertical plane. The measurement of the angles of the rods was done in the same way as described by de Renzi et al. ${ }^{3}$ The same applies to the test positions of the model in the five trials.

In the line orientation test the response-choice display consisted of an array of lines numbered 1 to 11 , each separated by an angle of $18^{\circ}$. Each stimulus consisted of two lines that represented the proximal, middle or distal one half $(1.9 \mathrm{~cm})$ of a response-choice line $(3.8 \mathrm{~cm}) .^{2}$ Five practice items employing the full-length lines preceded the test items. The subjects were informed whether or not their responses were correct on the practice items but not on the test items. The task was to indicate by pointing or naming the two lines in the response-choice display that had the 
Table 1 Site of the lesion

\begin{tabular}{lll}
\hline $\begin{array}{c}\text { Pre- and post- } \\
\text { rolandic lesion }\end{array}$ & fronto-parietal & 2 \\
\hline temporo-parietal & 6 \\
\hline $\begin{array}{c}\text { Exclusively post- } \\
\text { rolandic lesion }\end{array}$ & $\begin{array}{c}\text { temporo-parieto- } \\
\text { occipital } \\
\text { parieto-occipital }\end{array}$ & 2 \\
occipital & 3 \\
\hline
\end{tabular}

Table 2 Neurological signs two weeks and six months after the stroke in 16 patients with a posterior lesion of the right hemisphere, who underwent sequential tests of spatial function

\begin{tabular}{llll}
\hline & & \multicolumn{2}{c}{ Right hemisphere } \\
\cline { 3 - 4 } & & 2 weeks & 6 months \\
\hline Slight weakness & $\begin{array}{l}\text { arm } \\
\text { leg } \\
\text { both }\end{array}$ & 7 & 4 \\
Moderate weakness & $\begin{array}{l}\text { arm } \\
\text { leg } \\
\text { both }\end{array}$ & 1 & 2 \\
Severe weakness & $\begin{array}{l}\text { arm } \\
\text { leg } \\
\text { both }\end{array}$ & 4 & \\
Sensory impairment & 12 & 4 \\
Isolated visual field defect & 4 & 1 \\
No neurological deficit & & & \\
\hline
\end{tabular}

same angle (pointed in the same direction) and occupied the same location as the two stimulus lines. No time limit was imposed. The material was assembled in booklet form (30 items) so that when opened crosswise the stimulus appeared on the top page and the response-choice display on the bottom page of the booklet. Responses were scored as correct if both the stimulus lines were accurately identified.

\section{Assessment of lesion size}

In order to investigate a possible correlation between the size of the lesion and the speed of recovery a simple procedure was devised for assessing the size of the lesion. With a planimeter (the Ott polar planimeter) the surfaces of the lesion were measured on all the sections of the CT scan upon which it was visible. The planimeter gives the surface in nonius units. In order to get the actual size of the surface of the lesion in square centimetres, the measurement in nonius units had to be multiplied by a certain figure. The reduction factor of the photograph is included in the calculation of this figure. I assumed that the shape of an infarct approximates that of a double cone with its axis at right angles to the plane of scanning. By this assumption the volume could be calculated by taking the product of the distance between the sections and the sum of the surface of all sections. This is easy to see from an example. If the lesion is visible on three CT scan sections and the surfaces of the lesion on these sections are $A, B$ and $C$ respectively, and the distance between two sections is $h$, then the volume can be calculated with the following formula

$$
\begin{aligned}
V=1 / 2 A h+1 / 2(A+B) h+ & 1 / 2(B+C) h \\
& +1 / 2 C h=h(A+B+C) .
\end{aligned}
$$

In fresh infarcts the margins can be blurred. So they were chosen to include only marked hypodensity in order to reduce this inaccuracy. Each surface was measured three times and the average was calculated. (The difference between the highest and the lowest sum of the volumes was $2 \cdot 6 \%$.)

\section{Results}

Figures 1 and 2 each show the time course of the mean error scores for the eight patients with a preand post-rolandic extension of the lesion and the eight patients with an exclusively post-rolandic lesion of the right hemisphere, respectively. The results for the rod orientation test are shown above the abscissa and those for the line orientation test below it. On the abscissa the follow-up period is indicated in months. The scores on the line orientation test have been corrected for age and sex. ${ }^{2}$ For the latter test there were only three patients with a defective performance. All three scored severely defective.

For the rod orientation test there were four mean error rates for both the visual and the tactile part of the test: horizontal error, vertical error, model left or right. As there were no significant differences in error rates according to the site of presentation of the model in these two subgroups of brain-damaged patients, further statistical analysis was done on the mean of all error rates per individual.

For the statistical assessment of changes an analysis of variance was used. Six months after the stroke a significant change in performance had occurred in the two groups as a whole for the visual as well as for the tactile part of the test $(p<0 \cdot 001)$.

In comparing the test results of these patients six months after the stroke with those of the control

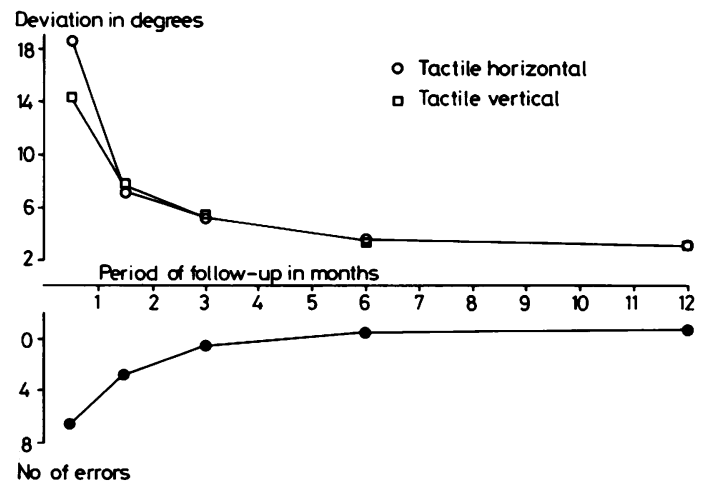

Fig 1 Course of the mean error scores of eight patients with a pre-and post-rolandic extension of the right-hemisphere lesion, for the rod orientation test above the abscissa and for the line orientation test below it. 


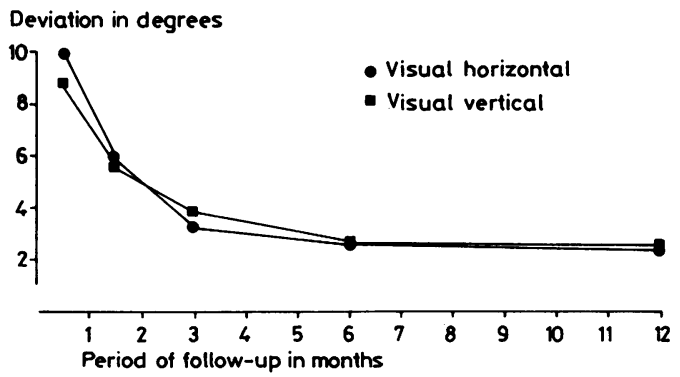

Deviation in degrees

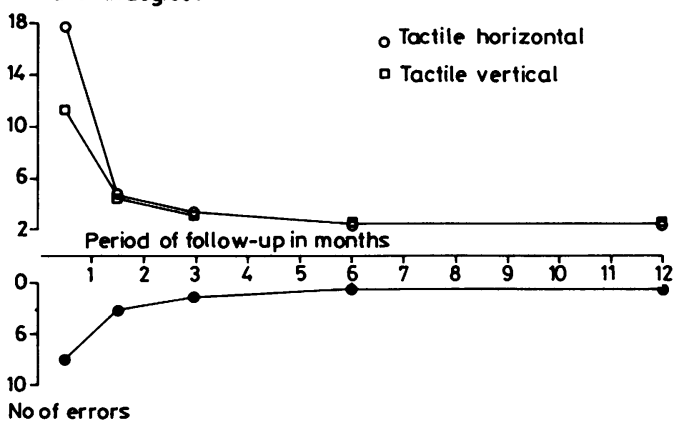

Fig 2 Course of the mean error scores of eight patients with an exclusively post-rolandic lesion of the right hemisphere, for the rod orientation test above the abscissa and for the line orientation test below it.

group, no statistically significant difference was found. The recovery on the rod orientation test was paralleled by the neurological improvement (table 2).

Only one patient had an apraxia for dressing at the initial examination, which had disappeared after four weeks.

In the patient group with an exclusively postrolandic lesion one patient still had a defective performance on the visual as well as on the tactile part of the test both six months and one year after the stroke.

Figure 3 shows the relation between the volume of the lesion and the recovery time. A patient was considered to have recovered, for the purpose of this study, when the error rates became equal to the mean error rates of the adult control group plus twice the standard deviation. Although there was no linear relationship, some form of relation existed.

The lesions of the patients with an exclusively post-rolandic lesion had an area in common. (a) On the slice at the level of the cella media: the white matter between the lateral occipital gyrus and the parieto-occipital sulcus; (b) on the slice below the previous one: the white matter between the lateral occipital gyrus, middle temporal gyrus and the
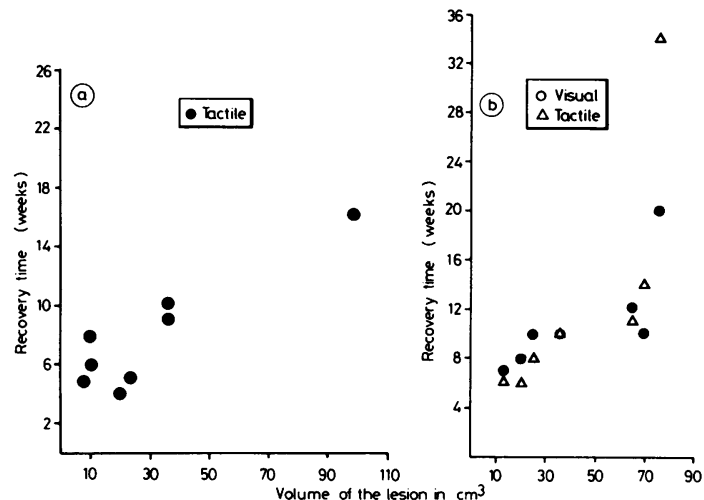

Fig 3 (a)Relation between the size of the lesion and the recovery time in the patient group with a pre- and postrolandic extension of the lesion. (b) Relation between the size of the lesion and the recovery time in the patient group with an exclusively post-rolandic lesion.

parieto-occipital sulcus. This area overlapped the common area described in a previous article. ${ }^{1}$

\section{Discussion}

Most patients performed again at a normal level between three and six months after the stroke. It is striking that in a previous study ${ }^{1}$ patients with a posterior lesion of the right hemisphere performed significantly worse than control patients one year after the stroke, which was not found in the present investigation. In terms of individual patients, this difference in findings is reflected by the fact that in the previous study five patients only partly recovered, whereas in the present group only one patient showed a partial recovery. The difference found cannot be explained on the basis of a difference in lesion size, because the mean volume of the infarct was $29 \mathrm{~cm}^{3}$ in the first study ${ }^{1}$ and $36 \mathrm{~cm}^{3}$ in the present group. The most probable explanation for the improvement in performance in the present group is training. They were tested five times while the patients in the first group were only tested twice. ${ }^{1}$

The results show that the line orientation test was much less sensitive in assessing spatial disorientation than the rod orientation test: only three patients had a defective performance with the line orientation test. However, all patients had a disturbance in spatial perception when examined with the rod orientation test, those with a pre- and post-rolandic extension of the lesion principally on the tactile modality, and those with an exclusively post-rolandic lesion on both modalities tested. Since the line orientation test can be presented only by visual means, no failures were expected on this test in the first group of 
patients. Accordingly, Benton et $a^{2}$ had no failures in their patient group with a peri-rolandic lesion.

Of the three patients with a disturbed performance on the line orientation test, one had a preand post-rolandic extension of the lesion, and two an exclusively post-rolandic lesion. All three scored "severely defective". In two patients this defective performance was found only at the test two weeks after the stroke. In one patient the defective performance continued for 22 weeks, which paralleled the recovery of spatial disorientation as measured with the rod orientation test.

There are several possible explanations for the difference in results with the line orientation test in this investigation and in the study done by Benton et al. ${ }^{2} 1$. Aetiology: only infarcts were included in this study; vascular, neoplasm and other brain lesions occurred in theirs. 2. The presence or absence of oedema or a midline shift could influence the test results. These data are not mentioned in their article. 3. A low sensitivity of the line orientation test. Finally a crude relationship between the size of the lesion and the speed of recovery was found.

\section{References}

${ }^{1}$ Meerwaldt JD, Harskamp F van. Spatial disorientation in right-hemisphere infarction. $J$ Neurol Neurosurg Psychiatry 1982;45:586-90.

${ }^{2}$ Benton AL, Varney NR, Hamsher K d S. Visuospatial Judgment. Arch Neurol 1978;35:364-7.

${ }^{3}$ De Renzi E, Faglioni P, Scotti G. Judgment of spatial orientation in patients with focal brain damage. J Neurol Neurosurg Psychiatry 1971;34:489-95. 